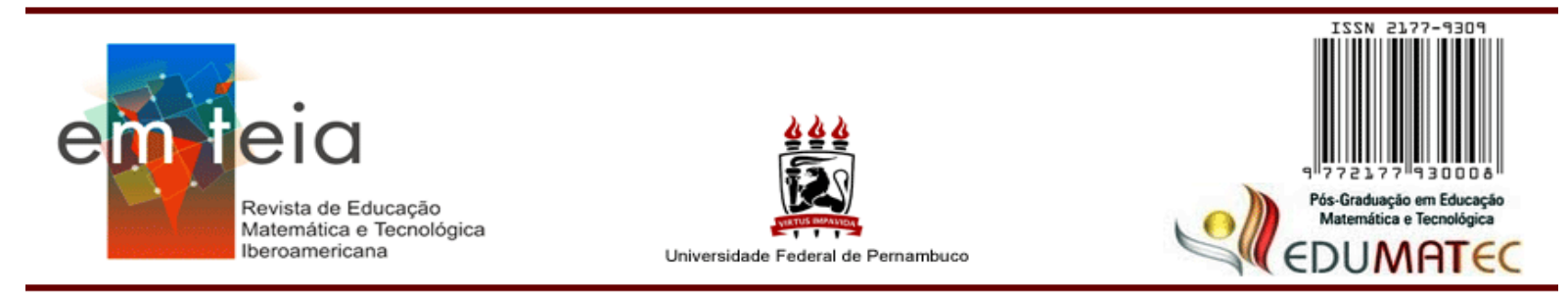

\title{
ANÁLISE SISTEMÁTICA DO MÉTODO EMPÍRICO DE MEDIÇÃO DE TERRAS
}

\author{
SYSTEMATIC ANALYSIS OF THE EMPIRICAL LAND MEASUREMENT \\ METHOD
}

\author{
Ednaldo Hermes da Silva \\ ednaldo.silva@univasf.edu.br \\ Mestre em Matemática \\ Universidade Federal do São Francisco \\ http://orcid.org/0000-0002-3084-690X \\ Erinaldo Ferreira do Nascimento \\ erigremio.2013@gmail.com \\ Licenciado em Matemática \\ Escola Municipal Pedro Lopes da Silva \\ https://orcid.org/0000-0003-2321-7854
}

\section{Resumo}

Este artigo discorre sobre a importância do conhecimento empírico para povos de zonas rurais, através da utilização desse tipo de conhecimento no cálculo de área de terras. O trabalho tem como objetivo analisar os processos matemáticos desenvolvidos por pequenos agricultores no cálculo de áreas de terras e a associação desse conhecimento com métodos sistemáticos já consagrados na literatura matemática. Apresenta uma abordagem qualitativa, um estudo de caso com pequenos agricultores inseridos em uma comunidade rural do município de Filadélfia/Ba, e teve como instrumento de coleta de dados um questionário composto por seis questões. Os dados obtidos mostram que o procedimento empírico é valido para o cálculo da área dos quadriláteros e seus resultados forneceram medidas exatas ou aproximadas, onde suas variações dependeram do formato da figura geométrica que representa a região em questão, sendo tanto mais próximas do valor real quanto mais regular for o quadrilátero.

Palavras-chave: Método empírico. Cubação de terras. Medidas padronizadas. Quadriláteros. Agricultores.

\footnotetext{
Abstract

This article discusses the importance of empirical knowledge for people in rural areas, through the use of this type of knowledge in the calculation of land area. The work aims to analyze the mathematical processes developed by small farmers in the calculation of land areas and the association of this knowledge with systematic methods already established in the mathematical literature. It presents a qualitative approach, a case study with small farmers inserted in a rural community in the city of Filadélfia/Ba and had as a data collection instrument a questionnaire composed of six questions. The data obtained show that the empirical procedure is valid for the calculation of the quadrilaterals and its results provided exact or approximate measurements, where its variations depended on the shape of the geometrical figure that represents the region in question, being both closer to the real value the more
} 
regular is the quadrilateral.

Keywords: Empirical method. Land cubation. Standardized measures. Quadrilaterals. Farmers. Introdução

A matemática é uma ciência viva, presente em todos os espaços em que o homem se encontra. Também está em constante evolução, contribuindo para o desenvolvimento sociocultural em todos os níveis do conhecimento e em diferentes culturas, desde as pequenas comunidades rurais aos grandes centros urbanos. Do ponto de vista do conhecimento sistemático, ela se interliga com todos os campos do conhecimento científico.

O desenvolvimento do conhecimento matemático no meio rural é realizado de diversas maneiras, sendo que a mais praticada é constituída por fatores culturais deixados de pais para filhos, de uma geração para outra. Pode-se dizer também que os aspectos culturais interferem e mantêm esses procedimentos matemáticos em continuidade. Para Rosa Neto (2002, p. 7 e 19), “A matemática foi criada e vem sendo desenvolvida pelo homem em função de suas necessidades. (...) A cultura é uma forma de adaptação porque é uma forma de atuação sobre o ambiente que foi construído juntamente com ela".

Nesse cenário de herança do conhecimento matemático, é cabível perceber o quanto a matemática vem sendo praticada em diferentes tempos e culturas, se afirmando como essencial para a existência da humanidade. Este trabalho consiste em fazer um estudo da aplicação do conhecimento matemático por agricultores no cálculo de medidas de propriedades rurais, enfatizada por eles como cubação de terra. A cubação é um método tradicional de medição de terra com importância na economia e no meio social, contribuindo para a conservação e a produção das pequenas propriedades do meio rural, sofrendo variações em cada cultura e região do país.

Os conhecimentos matemáticos surgiram a partir da necessidade das pequenas e grandes sociedades e foram adaptados em outras de acordo com a realidade de cada povo. Então, com essas adaptações matemáticas em cada cultura, os agricultores em estudo utilizam no cálculo de áreas de figuras planas (cubação de terra) sistemas de medidas não padronizadas que têm como instrumento de comprimento a braça, e por unidade de área, a tarefa. Para fundamentar o cálculo, utilizam-se as quatros operações básicas da matemática: adição, subtração, multiplicação e divisão.

A técnica utilizada para o cálculo das propriedades rurais nem sempre é aprendida na escola, visto que não consiste de um método formal de cálculo de áreas, além do que, muitos dos que dominam a técnica sequer frequentaram a escola. Esse método utilizado pelos 
agricultores advém de conhecimento adquirido por experiência, denominado de conhecimento empírico:

A matemática empírica se caracteriza como uma coleção de problemas (exemplos) concretos e práticos associados a atividades práticas como transações comerciais, administração, agricultura, etc... para os quais se apresentam soluções, aproximações e ou cálculos elaborados em termos de valores numéricos específicos (MIRANDA, 2009, p. 01).

Nesse contexto, este trabalho faz uma análise desse método prático de calcular quadriláteros usado pelos agricultores há gerações. Com base no procedimento dos cálculos desenvolvidos pelos agricultores, os resultados obtidos foram comparados com o processo sistemático de cálculo de áreas, a fim de verificar se os resultados obtidos através dos dois métodos são condizentes.

Fazendo um apanhado da estrutura macro e afunilando as questões envolvidas para se chegar a um resultado condizente e mostrar o quanto o conhecimento matemático contribui para o desenvolvimento de uma sociedade é inerente analisar todos os aspectos envolvidos no processo de construção de um método, e traçar metas científicas para provar que o fato é verídico. Assim, o presente artigo se objetiva em analisar os processos matemáticos desenvolvidos por pequenos agricultores para o cálculo de áreas de figuras planas, para obtenção das tarefas de terras e analisar os procedimentos empíricos no decorrer da apuração do resultado matemático.

Os procedimentos empíricos analisados fazem parte da construção e desenvolvimento dos saberes matemáticos, sobretudo na realização dos métodos de cálculo de áreas (cubação de terras) dos pequenos agricultores, inseridos no território de identidade quilombola pertencente à comunidade de aguadas na zona rural do município de Filadélfia/Ba.

\section{Metodologia}

Optamos pela realização de uma pesquisa com abordagem qualitativa, pois esse tipo de metodologia permite que o pesquisador tenha contato direto com os sujeitos envolvidos. Para Minayo (2001), a pesquisa qualitativa trabalha com o universo de significados, motivos, aspirações, crenças, valores e atitudes, o que corresponde a um espaço mais profundo das relações, dos processos e dos fenômenos que não podem ser reduzidos à operacionalização de variáveis. 
Concebe-se que o tal método permite uma compreensão básica e se enquadra nos aspectos de procedimento um valor intrínseco da realidade multidimensional e historicamente situada como estudo de caso:

Um estudo de caso pode ser caracterizado como um estudo de uma entidade bem definida como um programa, uma instituição, um sistema educativo, uma pessoa, ou uma unidade social. Visa conhecer em profundidade o como e o porquê de uma determinada situação que se supõe ser única em muitos aspectos, procurando descobrir o que há nela de mais essencial e característico (GIL, 2007, p. 54).

A abordagem em pauta contribui para alcançar os objetivos propostos dentro de uma determinada pesquisa, facilitando o diálogo direto com os sujeitos envolvidos, chegando aos resultados do método em estudo.

Os sujeitos envolvidos no processo de construção dessa pesquisa foram pequenos produtores rurais, situados na comunidade de aguadas no município de Filadélfia/Ba. Utilizouse para a coleta de dados um questionário com seis perguntas, sendo cinco de múltipla escolha e uma aberta.

O questionário, segundo Gil (1999, p. 128), pode ser definido "como a técnica de investigação composta por um número mais ou menos elevado de questões apresentadas por escrito às pessoas, tendo por objetivo o conhecimento de opiniões, crenças, sentimentos, interesses, expectativas, situações vivenciadas etc."

Sendo escolhidos três produtores para participar da pesquisa, e, para resguardar a identidade dos participantes, optamos em identificarmos por AGR-01, AGR-02 e AGR-03 e assim serão chamados no decorrer desse trabalho. Os entrevistados possuem faixa etária de idade entre trinta (30) anos e setenta (70) anos, todos são filhos de produtor, um com o ensino médio incompleto e dois com ensino fundamental incompleto.

$\mathrm{Na}$ construção do questionário foi levado em conta agentes reais que fazem parte da cultura local no desenvolvimento da aprendizagem matemática, e que se tornaram essenciais para a coleta de dados.

\section{Fundamentação Teórica}

O referencial teórico da pesquisa foi estruturado nos três tópicos: História da geometria, Aplicação do cálculo no meio rural e Medidas padronizadas e não padronizadas.

História da geometria 
A geometria sempre esteve atrelada ao desenvolvimento humano, tendo sido elaborada e usada dentro do contexto social. A própria palavra geometria, que deriva do grego "medir a terra", parece coincidir com as necessidades do dia a dia. Partilhar terras férteis às margens dos rios, construir casas, observar e prever os movimentos dos astros, são algumas das muitas atividades humanas que sempre dependeram de operações geométricas. Documentos sobre as antigas civilizações egípcia e babilônica comprovam bons conhecimentos do assunto, geralmente ligados à medição de terras e à astrologia.

Para Eves (2004), o Rio Nilo, conhecido como a fonte de vida do Egito a milhares de anos, deu origem aos primeiros sinais da matemática, quando os povos abandonaram a vida nômade de até então e começaram a fixar suas residências ao redor deste rio, que proporcionava condições perfeitas para a agricultura.

Sobre os primeiros sinais da matemática, o famoso historiador grego, Heródoto, do Século V a.C., certifica que

Esse faraó (Sesótris) realizou a partilha das terras, concedendo a cada egípcio uma porção igual, com a condição de ser-lhe pago todos os 3 anos certo tributo; se o rio carregava alguma parte do lote de alguém, o prejudicado ia procurar o rei e expor-lhe o ocorrido. O soberano enviava agrimensores para o local, para determinar a redução sofrida pelo terreno, passando o proprietário a pagar um tributo proporcional ao que restara. Eis, ao que me parece, a origem da geometria, que teria passado do Egito para a Grécia (GARBI, 2008, p. 12).

Note-se em seguida na fala de D’Ambrósio:

A Geometria, na sua origem e no próprio nome, está relacionada com as medições de terreno. Como nos conta Heródoto, a geometria foi aprendida dos egípcios, onde era mais que uma simples medição de terreno, tendo tudo a ver com o sistema de taxação de áreas produtivas (D’AMBRÓSIO, 2005, p. 36).

Ainda sobre o desenvolvimento da geometria, Oliveira (2012) afirma que a mesma teve suas origens ligadas às necessidades do homem no seu dia a dia, pois havia a necessidade de medir as terras para repartir as partes férteis que ficavam às margens do rio.

Os babilônios parecem ter conhecido as regras gerais de medição de áreas e volumes. Por exemplo, mediram a circunferência de um círculo como três vezes o diâmetro e a área como um doze avos do quadrado da circunferência, o que seria correto se $\pi$ fosse estimado como 3 .

A geometria babilônica se relaciona intimamente com a mensuração prática. De numerosos exemplos concretos infere-se que os babilônicos [...] deviam estar familiarizados com as regras gerais da área do retângulo, da área do triângulo retângulo, e do triangulo isósceles (e talvez da área de um triângulo genérico), da área de um trapézio retângulo, do volume de um paralelepípedo reto-retângulo e, mais geralmente do volume de um prisma reto de base trapezoidal... (EVES, 2011, p. 61). 
Convém considerar, no entanto, que o desenvolvimento geométrico é de extrema importância para toda a sociedade, em especial, para os que residem no meio rural, pois, necessitam das representações de suas figuras planas para calcular as áreas das propriedades rurais que são conhecidas como tarefas de terras. Por estes motivos talvez justifique a origem da sua palavra "geometria", derivada do grego geo, que significa terra, e metria, que significa medida, assim significando "medição de terra".

Aplicação do cálculo no meio rural

A aplicação da geometria e do conceito de área é algo indispensável na atual sociedade. Para Leonardo (2010), encontramos a geometria plana como uma parte da matemática que tem maior utilização no nosso dia a dia, pois precisamos calcular medidas de comprimento, área de lugares, distâncias entre pontos etc.

As figuras geométricas são a base para as representações do conteúdo de geometria, pois é por meio delas que conseguimos realizar alguns cálculos impostos em nossa sociedade, que são chamadas de medidas. As medidas surgiram e se adaptaram a cada civilização, e são utilizadas no meio rural para o cálculo de áreas em propriedades rurais, denominadas pelos que residem nesse meio como tarefas.

É usada como uma medida agrária por diversos agricultores, a medição de um terreno em tarefas ou cubação de terras como é empregado por vários agricultores do interior de alguns estados brasileiros é uma das formas de representar a área de uma propriedade em uma unidade de medida diferente das convencionais, mas que para eles esta medida é bastante comum (SILVA, 2016, p. 75).

É importante destacar que o método adotado no meio rural para o cálculo de áreas geralmente não é um conhecimento adquirido na escola, mas adquirido por meio de experiências que passam através de gerações. É o que chamamos de conhecimento empírico. Empírico é aquele conhecimento adquirido durante toda a vida, no dia a dia, que não tem comprovação científica. Tartuce (2006) traz uma reflexão relacionada a esse tipo de conhecimento:

É o conhecimento obtido ao acaso, após inúmeras tentativas, ou seja, o conhecimento adquirido através de ações não planejadas. É o conhecimento do dia a dia, que se obtém pela experiência cotidiana. É espontâneo, focalista, sendo por isso considerado incompleto, carente de objetividade. Ocorre por meio do relacionamento diário do homem com as coisas. Não há a intenção e a preocupação de atingir o que o objeto contém além das aparências. Fundamentado apenas na experiência, doutrina ou atitude, que admite quanto à origem do conhecimento de que este provenha apenas da experiência (TARTUCE, 2006, p. 6). 
Medidas padronizadas e medidas não-padronizadas

Diferentes povos tiveram suas unidades de medidas próprias, as quais eram definidas de maneira arbitrária e variavam de um país para outro, dificultando as transações comerciais e o intercâmbio científico entre eles. O palmo, o passo, a braça, a polegada são exemplos de antigas unidades de medir comprimentos que utilizam as partes do corpo humano, e que ainda hoje são usadas em muitas situações.

Com a revolução industrial e a maior interação entre povos, essa diferença entre unidades de medida tornou-se um problema para as relações comerciais. Muitas discussões aconteceram na tentativa de contornar esse problema, até que em 1960 em Paris, cientistas se reuniram para determinar unidades de medidas que pudessem ser usadas em todo o mundo. Assim, em 1960, surgiu o sistema SI, Sistema Internacional de Unidades, visando uniformizar e facilitar as medições e as relações internacionais daí decorrentes. No SI, a unidade de medida padrão de comprimento utilizada com maior frequência é o metro, que tem mais duas vertentes, o metro quadrado $\left(\mathrm{m}^{2}\right)$, que é referência para o cálculo de áreas, e o metro cubico $\left(\mathrm{m}^{3}\right)$ que é direcionado ao cálculo de volume; todos são utilizados na sociedade contemporânea para medir essas grandezas associadas a objetos.

No entanto, muitas pessoas ao redor do mundo ainda utilizam unidades de medidas próprias. A polegada, libra, milhas, por exemplo, continuam sendo usadas ainda hoje, para medir objetos, distâncias náuticas, massas. A braça e a tarefa são outro exemplo de antigas unidades de medida, ainda largamente usadas e compreendidas por muitos trabalhadores rurais e outras pessoas envolvidas com o meio rural.

A braça e a tarefa, bem como suas relações entre o metro e o metro quadrado, são descritas abaixo. Temos:

01 braça (comprimento) $=2,20 \mathrm{~m}$

01 tarefa $($ comprimento $)=30$ braças $=66,00 \mathrm{~m}$

01 tarefa (área) $=900$ braças $^{2}=4356 \mathrm{~m}^{2}$

É tradição no meio rural, os agricultores usarem a grandeza tarefa para descrever comprimento ou área. Neste trabalho, o contexto, ou uma observação específica, deixará claro tratar-se de um caso ou outro.

\section{Desenvolvimento}

O método de cálculo usado pelos agricultores 
O formato geométrico predominante dos espaços de terra que os agricultores se deparam e têm a necessidade de calcular são os quadriláteros. Na ausência de uma maneira sistemática, ou fórmula matemática, para calcular essas regiões, eles lançam mão de uma técnica primitiva e bastante pragmática. Nesta seção, faremos a descrição do método usado pelos agricultores, a fim de que possamos fazer a análise sistemática desse procedimento mais adiante.

Considere um quadrilátero convexo qualquer, como o da figura 01 abaixo.

Figura 1: Quadrilátero $A B C D$

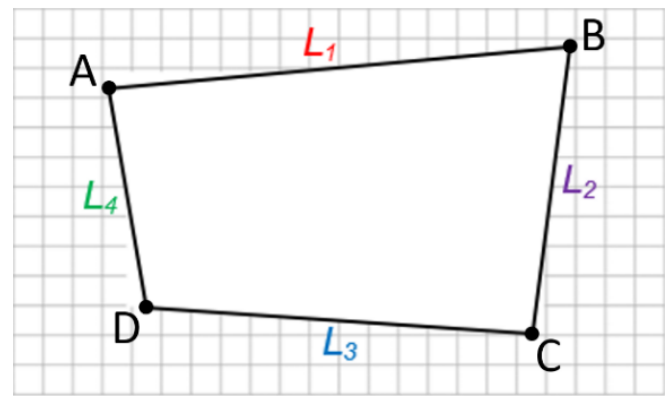

Fonte: Os autores

Suponhamos que $L_{1}$ e $L_{3}$ são as medidas dos lados maiores $\mathrm{AB}$ e CD, respectivamente, $L_{2}$ e $L_{4}$ as medidas dos lados menores, $\mathrm{BC}$ e $\mathrm{AD}$, respectivamente. Para calcular a área da região interna a esse quadrilátero, os agricultores procedem da seguinte forma:

Multiplicam-se dois resultados: a soma das medidas dos dois lados maiores e a soma das medidas dos dois lados menores, dividindo-se em seguida o resultado por 4. Assim:

$$
A=\frac{(\mathrm{AB}+\mathrm{CD}) \cdot(\mathrm{AD}+\mathrm{BC})}{4}
$$

Vale ressaltar que a fórmula dada pela expressão 01 é colocada aqui pelos autores. $\mathrm{O}$ cálculo feito pelos agricultores é desenvolvido de forma prática, herdado por tradição, e é feito utilizando os valores numéricos das medidas do terreno.

Como essa fórmula não existe na literatura matemática, vamos mostrar que ela é equivalente a outra que pode ser obtida decompondo um quadrilátero em quatro triângulos. Isso tem como objetivo fazer a comparação do método empírico com os valores sistemáticos.

Organização e formalização do método empírico

Suponha que se queira calcular a área tal qual a representada no quadrilátero $\mathrm{ABCD}$ como na figura 2, de medidas das arestas $\mathrm{x}, \mathrm{y}, \mathrm{z}$ e w. 


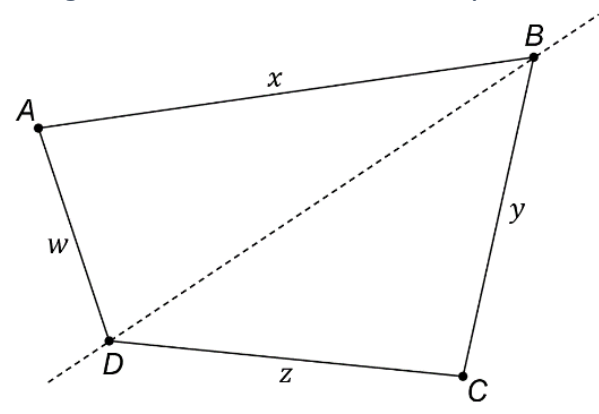

Fonte: Os autores

Ainda antes de explicação da expressão 01 para cada tipo de quadrilátero, vamos separar o tipo de quadrilátero recai em dois casos, de acordo com as medidas dos lados:

\section{Caso 1: Os dois lados com medidas maiores são lados opostos}

Considerando a figura 2, suponhamos que os dois maiores lados sejam $\mathrm{AB}$ e DC, opostos no quadrilátero. Neste caso, é só aplicar o procedimento empírico, descrito pela fórmula da expressão 01, e teremos a área experimental.

\section{Caso 2: Os dois lados com medidas maiores são lados adjacentes}

Suponhamos que os dois maiores lados sejam AB e BC na figura 2. Para melhor organização, faremos um procedimento geométrico, que consiste em deixar os lados maiores de lados opostos, tal qual no Caso 1, descrito anteriormente.

Suponha que os dois maiores lados sejam os lados $\mathrm{AB}$ e $\mathrm{BC}$, de medidas $x$ e $y$. (figura 3-a). Primeiro, separamos o quadrilátero $\mathrm{ABCD}$ em dois triângulos: $\mathrm{ABD}$ e B'CD'. (figura 3b). A seguir fazemos uma "rotação espacial" do triângulo B'CD', sem alterar suas formas (figura 3-c). Assim, teremos uma nova maneira de acoplar os triângulos $\mathrm{ABC}$ e $\mathrm{B}^{\prime} \mathrm{CD}^{\prime}$ para formar um novo quadrilátero (figura 4). Observe que este novo quadrilátero possui a mesma área que a do quadrilátero inicial. 


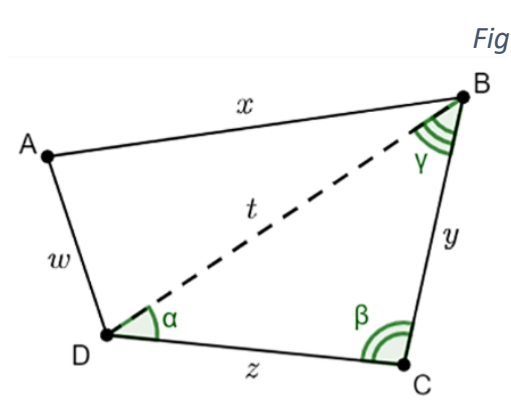

(a)

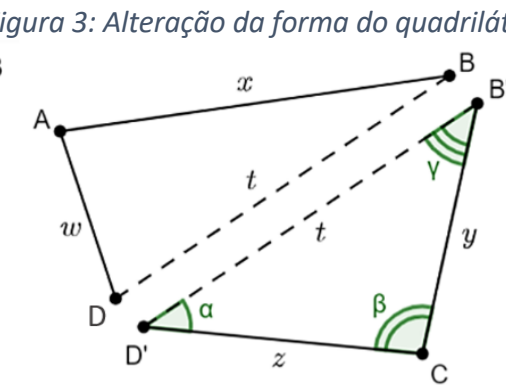

(b)

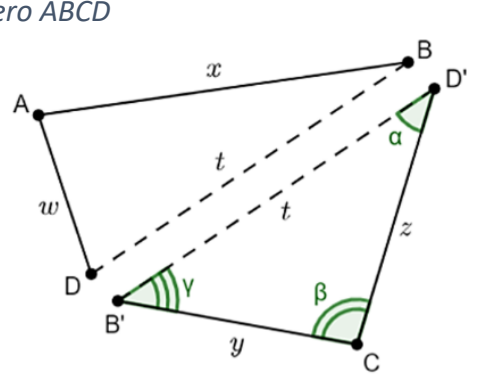

(c)

Fonte: Os autores

Vale ressaltar que esse é um procedimento matemático abstrato, e tem a função única e exclusiva de deixar os dois maiores lados opostos no quadrilátero. Tudo isso para que possamos fazer a análise sistemática, de acordo com a expressão 02 , obtida a seguir.

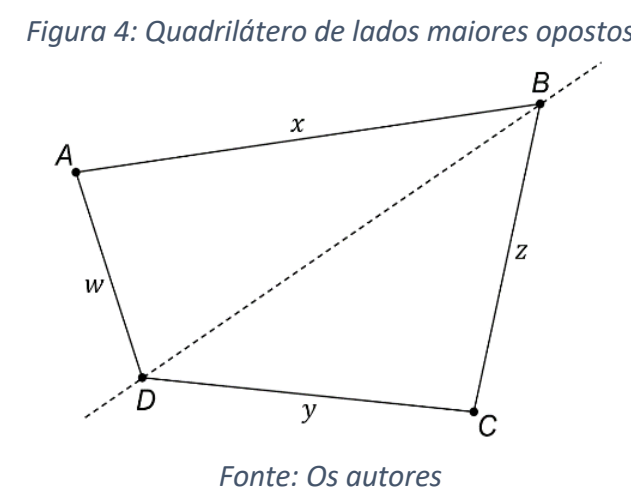

Observe agora que, independente da posição os dois maiores lados no quadrilátero, para o cálculo da área, podemos considerar sempre de lados opostos. Agora, podemos continuar com a explicação da expressão 01. Então:

$$
\begin{aligned}
A & =\frac{(\mathrm{AB}+\mathrm{CD}) \cdot(\mathrm{AD}+\mathrm{BC})}{4} \\
& \Leftrightarrow \frac{(\mathrm{AB}+\mathrm{CD})}{2} \cdot \frac{(\mathrm{AD}+\mathrm{BC})}{2} \\
& \Leftrightarrow\left(\frac{\mathrm{AB}}{2}+\frac{\mathrm{CD}}{2}\right) \cdot\left(\frac{\mathrm{AD}}{2}+\frac{\mathrm{BC}}{2}\right) \\
& \Leftrightarrow \frac{\mathrm{AB} \cdot \mathrm{AD}}{4}+\frac{\mathrm{AB} \cdot \mathrm{BC}}{4}+\frac{\mathrm{CD} \cdot \mathrm{AD}}{4}+\frac{\mathrm{CD} \cdot \mathrm{BC}}{4}
\end{aligned}
$$

donde encontramos uma fórmula equivalente de obter o valor da área A:

$$
A=\frac{\mathrm{AB} \cdot \mathrm{AD}}{4}+\frac{\mathrm{AB} \cdot \mathrm{BC}}{4}+\frac{\mathrm{AD} \cdot \mathrm{CD}}{4}+\frac{\mathrm{BC} \cdot \mathrm{CD}}{4}
$$


Esta maneira de representar a fórmula utilizada pelos agricultores será útil para compará-la com a área efetiva, dada por métodos consagrados na literatura matemática.

Aplicação do experimento

Para verificar como agricultores procederiam com o método, bem como para conferir suas respostas, aplicamos o mesmo questionário com seis questões para os três agricultores, os quais denominamos por AGR-01, AGR-02 e AGR-03.

A primeira pergunta referiu-se à importância da matemática no desenvolvimento do campo, teve-se como resultado cem por cento (100\%) de afirmação positiva. A segunda relacionada ao nível de escolaridade, e teve-se as seguintes respostas, $(33,33 . . \%)$ com ensino fundamental anos iniciais incompleto, $(33,33 . . \%)$ com ensino fundamental anos finais incompleto e $(33,33 . . \%)$ com ensino médio incompleto. Na terceira pergunta questionamos o fator idade de cada participante, donde obtivemos que $70 \%$ está entre 35 a 50 anos e $30 \%$ entre 51 a 70 anos de idade. A quarta pergunta foi referente à unidade de medida utilizada para medir as propriedades rurais: $100 \%$ assinalaram a tarefa como unidade de medida e todos salientaram que a utilizam há décadas, pois foi deixada de pai para filhos. Na quinta questão, questionamos onde aprendeu o método, para o qual 30\% afirmaram que foi na escola, e $70 \%$ que não foi na escola. Por fim, na sexta pergunta propusemos um exemplo hipotético de mapa de um terreno a partir do qual eles precisavam medir a sua área.

Os agricultores foram entrevistados separadamente e resolveram, cada um deles, o mesmo problema hipotético de área. A seguir, temos a imagem dos três agricultores resolvendo o problema proposto:

Figura 5: Agricultores calculando o exemplo proposto

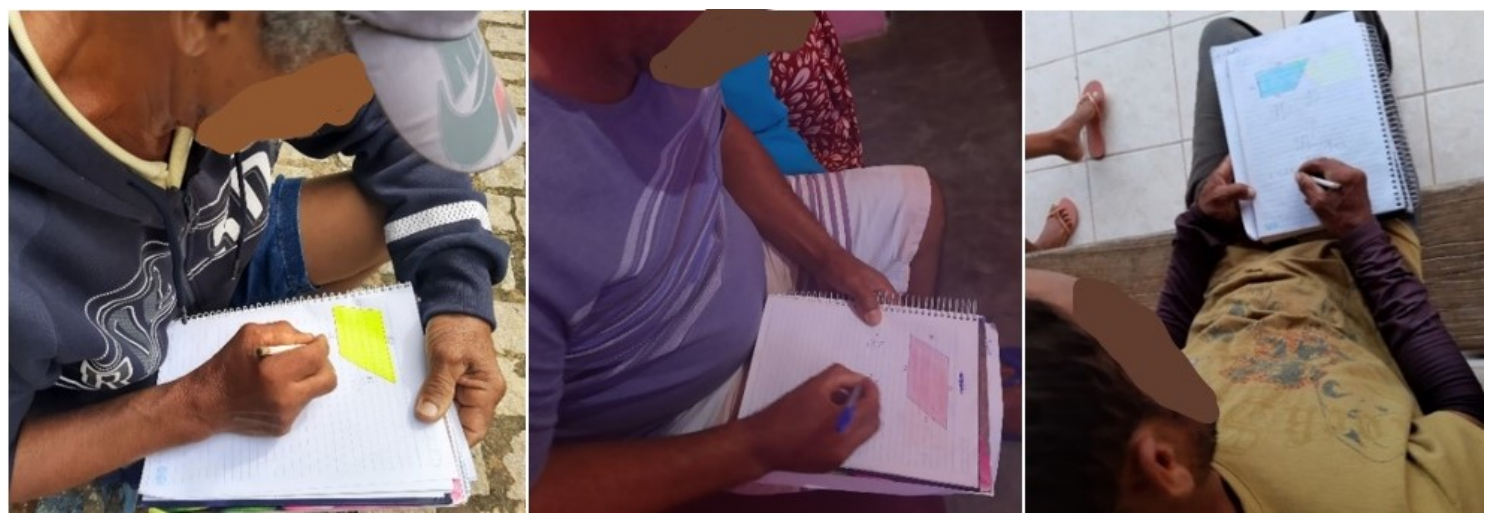

Fonte: Os autores 
Essa última pergunta buscou verificar o desenvolvimento do cálculo pelos participantes. Ao analisar, percebeu-se que todos encontraram a mesma resposta.

\section{Discussão dos resultados}

Análise do cálculo desenvolvido pelos agricultores

Faremos agora a análise das respostas dos agricultores para a sexta questão do questionário. Atribuímos a eles o cálculo da área de um terreno representado por um quadrilátero, cuja unidade de medidas dos lados foi dada em braças.

A figura 6 a seguir mostra o cálculo desenvolvido pelo AGR-01.

Figura 6: Resposta do AGR-01 para o problema proposto

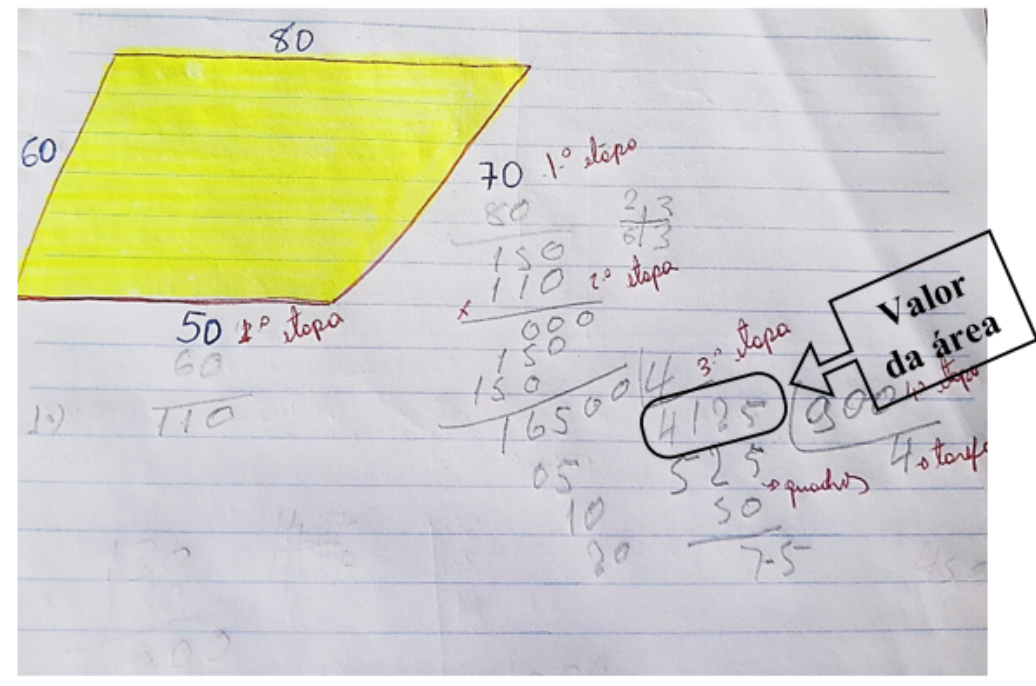

Fonte: Os autores

O AGR-01 lançou mão do método empírico, dados pela fórmula da expressão 01:

$$
\begin{aligned}
A & =\frac{(70+80) \times(50+60)}{4} \\
& \Leftrightarrow \frac{(150) \times(110)}{4} \\
& \Leftrightarrow \frac{(150) \times(110)}{4} \\
& \Leftrightarrow \frac{16500}{4} \\
& \Leftrightarrow 4125
\end{aligned}
$$

Esta medida é o número de braças quadradas. Uma vez que uma tarefa corresponde a 900 braças quadradas, dividiu-se em seguida o resultado que se obtivera por 900 , encontrando a medida em tarefas. 
Vemos que o agricultor somou as duas medidas maiores, somou as medidas menores, multiplicou os resultados e dividiu esse produto por 4, obtendo o valor 4125. Em seguida ele dividiu este valor por 900, obtendo a medida da área em tarefas como sendo 4. Ainda é possível perceber que ele obteve um resto na divisão, ao qual eles denominam de quadros. Mas este submúltiplo da tarefa não vem ao estudo nesse trabalho.

Para verificar que o procedimento usado pelo agricultor é o correspondente no desenvolvimento algébrico a partir de uma expressão usada como área, faremos aqui uma nova organização do cálculo, usando a expressão 02. Ressaltamos que estes cálculos não foram feitos pelos entrevistados. Podemos verificar que o procedimento adotado pelo agricultor é equivalente a usar o método dado pela fórmula 02 : tomando os lados 80, 70, 60 e 50 como sendo os lados $\mathrm{AB}, \mathrm{CD}, \mathrm{AD}$ e $\mathrm{BC}$, respectivamente. Então:

$$
\begin{aligned}
A & =\frac{\mathrm{AB} \cdot \mathrm{AD}}{4}+\frac{\mathrm{AB} \cdot \mathrm{BC}}{4}+\frac{\mathrm{AD} \cdot \mathrm{CD}}{4}+\frac{\mathrm{BC} \cdot \mathrm{CD}}{4} \\
& \Leftrightarrow \frac{80 \cdot 60}{4}+\frac{80 \cdot 50}{4}+\frac{60 \cdot 70}{4}+\frac{50 \cdot 70}{4} \\
& \Leftrightarrow \frac{80 \cdot 60}{4}+\frac{80 \cdot 50}{4}+\frac{60 \cdot 70}{4}+\frac{50 \cdot 70}{4} \\
& \Leftrightarrow 1200+1000+1050+875 \\
& \Leftrightarrow 4125
\end{aligned}
$$

Cuja resposta é igual ao método empírico adotado pelo AGR-01.

As mesmas respostas foram obtidas pelos agricultores 2 e 3 : 
Figura 7: Resposta do AGR-02 para o problema proposto

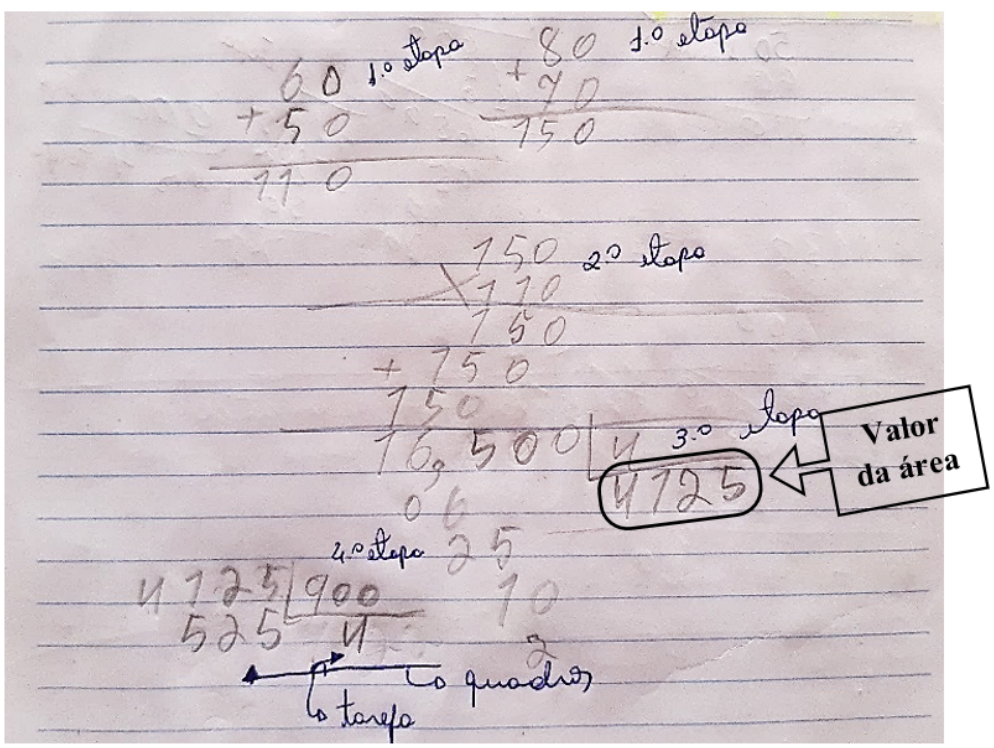

Fonte: Os autores

Figura 8: Resposta do AGR-03 para o problema proposto

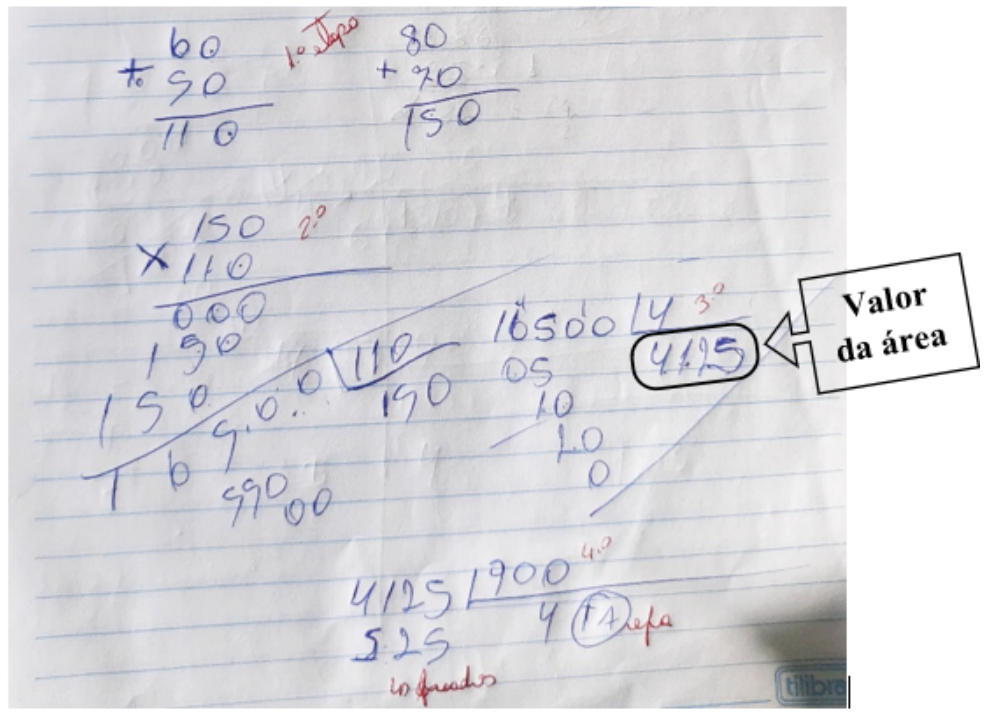

Fonte: Os autores

Análise sistemática do método empírico

O cálculo de áreas obtidos por esse método é exato apenas para os retângulos. Para os paralelogramos e demais quadriláteros, o método empírico fornece apenas valores aproximados. Neste trabalho, no entanto, não iremos mensurar essa diferença. Os procedimentos obtidos nos dão uma ideia de que existe uma aproximação, e que parece ser tão melhor, quanto mais regular for o quadrilátero.

Vamos separar a análise das áreas, de acordo com o tipo de quadriláteros. 


\section{Retângulo}

Suponha que se queira calcular a área de um terreno representado por um retângulo, tal qual o da figura 9 abaixo.

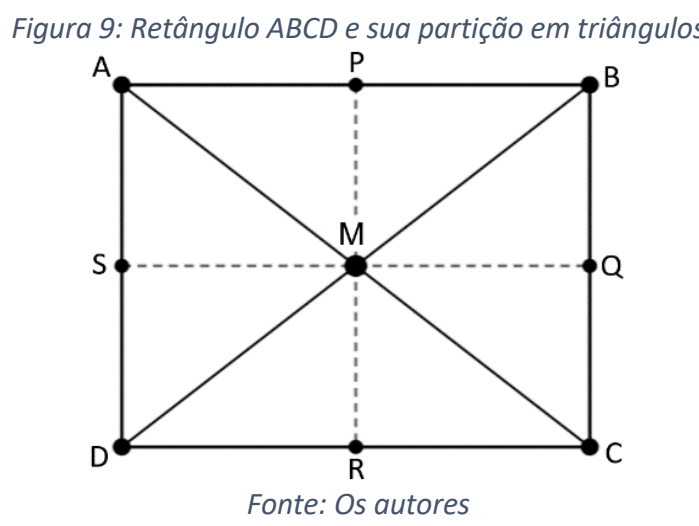

Inicialmente, tomamos os pontos médios $\mathrm{P}, \mathrm{R}, \mathrm{S}$ e $\mathrm{Q}$ dos respectivos lados $\mathrm{AB}, \mathrm{CD}, \mathrm{AD}$ e BC. Neste caso, os segmentos PR e SQ cruzam-se no ponto médio M. Assim:

$$
\begin{aligned}
& \mathrm{SM}=\mathrm{MQ}=\frac{\mathrm{AB}+\mathrm{CD}}{4} \\
& \mathrm{PM}=\mathrm{MR}=\frac{\mathrm{AD}+\mathrm{BC}}{4}
\end{aligned}
$$

A área do retângulo $\mathrm{ABCD}, A_{R}$, pode ser calculada somando-se as áreas dos triângulos AMB, CMD, AMD e BMC:

$A_{R}=\frac{\mathrm{AB} \cdot \mathrm{PM}}{2}+\frac{\mathrm{CD} \cdot \mathrm{MR}}{2}+\frac{\mathrm{AD} \cdot \mathrm{SM}}{2}+\frac{\mathrm{BC} \cdot \mathrm{MQ}}{2}$

Usando as expressões 03 e 04 :

$$
\begin{aligned}
A_{R} & =\frac{\mathrm{AB}}{2} \cdot \frac{\mathrm{AD}+\mathrm{BC}}{4}+\frac{\mathrm{CD}}{2} \cdot \frac{\mathrm{AD}+\mathrm{BC}}{4}+\frac{\mathrm{AD}}{2} \cdot \frac{\mathrm{AB}+\mathrm{CD}}{4}+\frac{\mathrm{BC}}{2} \cdot \frac{\mathrm{AB}+\mathrm{CD}}{4} \\
& \Leftrightarrow \frac{\mathrm{AB} \cdot \mathrm{AD}+\mathrm{AB} \cdot \mathrm{BC}}{8}+\frac{\mathrm{CD} \cdot \mathrm{AD}+\mathrm{CD} \cdot \mathrm{BC}}{8}+\frac{\mathrm{AD} \cdot \mathrm{AB}+\mathrm{AD} \cdot \mathrm{CD}}{8}+\frac{\mathrm{BC} \cdot \mathrm{AB}+\mathrm{BC} \cdot \mathrm{CD}}{8} \\
& \Leftrightarrow \frac{\mathrm{AB} \cdot \mathrm{AD}}{8}+\frac{\mathrm{AB} \cdot \mathrm{BC}}{8}+\frac{\mathrm{CD} \cdot \mathrm{AD}}{8}+\frac{\mathrm{CD} \cdot \mathrm{BC}}{8}+\frac{\mathrm{AD} \cdot \mathrm{AB}}{8}+\frac{\mathrm{AD} \cdot \mathrm{CD}}{8}+\frac{\mathrm{BC} \cdot \mathrm{AB}}{8}+\frac{\mathrm{BC} \cdot \mathrm{CD}}{8} \\
& \Leftrightarrow \frac{\mathrm{AB} \cdot \mathrm{AD}}{8}+\frac{\mathrm{AB} \cdot \mathrm{BC}}{8}+\frac{\mathrm{AD} \cdot \mathrm{CD}}{8}+\frac{\mathrm{BC} \cdot \mathrm{CD}}{8}+\frac{\mathrm{AB} \cdot \mathrm{AD}}{8}+\frac{\mathrm{AD} \cdot \mathrm{CD}}{8}+\frac{\mathrm{AB} \cdot \mathrm{BC}}{8}+\frac{\mathrm{BC} \cdot \mathrm{CD}}{8}
\end{aligned}
$$

Somando as parcelas semelhantes, encontramos a área do retângulo $A_{R}$, como 


$$
A_{R}=\frac{\mathrm{AB} \cdot \mathrm{AD}}{4}+\frac{\mathrm{AB} \cdot \mathrm{BC}}{4}+\frac{\mathrm{AD} \cdot \mathrm{CD}}{4}+\frac{\mathrm{BC} \cdot \mathrm{CD}}{4}
$$

donde obtemos a fórmula dada pela expressão 02 .

Assim, o cálculo desenvolvido para obter a expressão 05 foi feito através do procedimento geométrico sistemático. Esse resultado é igual à fórmula dada pela expressão 02 , o que nos permite concluir que a área obtida pelo método empírico é verdadeira para o caso de um retângulo.

\section{Paralelogramo}

Suponha agora que queiramos calcular a área de uma região interna ao paralelogramo, tal qual o da figura 10.

Inicialmente, tomamos os pontos médios $\mathrm{P}, \mathrm{R}, \mathrm{S}$ e $\mathrm{R}$ dos respectivos lados $\mathrm{AB}, \mathrm{CD}, \mathrm{AD}$ e BC. Os segmentos PR e SQ cruzam-se no ponto médio M.

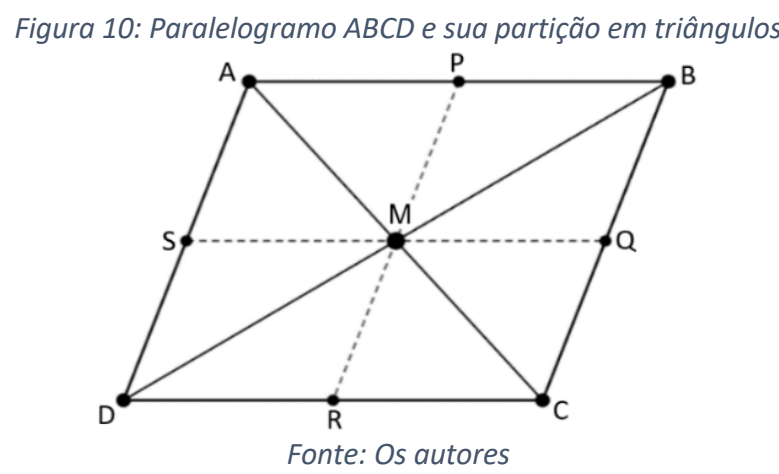

Para o paralelogramo, as relações dadas pelas expressões 03 e 04 também são verdadeiras, pois os lados são paralelos e congruentes.

Vamos calcular a área aproximada do paralelogramo $\mathrm{ABCD}$, somando-se as áreas dos triângulos AMB, CMD, AMD e BMC.

No entanto, uma vez que usaremos como "altura" desses triângulos, os segmentos PM, MR, SM e MQ, respectivamente, em vez de usar as alturas reais, EM, MG, HM e MF, iremos calcular uma área fictícia, pois as alturas usadas nesse cálculo são maiores que a altura real. (ver figura 11). Assim:

$A_{P}=\frac{\mathrm{AB} \cdot \mathrm{PM}}{2}+\frac{\mathrm{CD} \cdot \mathrm{MR}}{2}+\frac{\mathrm{AD} \cdot \mathrm{SM}}{2}+\frac{\mathrm{BC} \cdot \mathrm{MQ}}{2}$

Usando as expressões 03 e 04 :

$A_{P}=\frac{\mathrm{AB}}{2} \cdot \frac{\mathrm{AD}+\mathrm{BC}}{4}+\frac{\mathrm{CD}}{2} \cdot \frac{\mathrm{AD}+\mathrm{BC}}{4}+\frac{\mathrm{AD}}{2} \cdot \frac{\mathrm{AB}+\mathrm{CD}}{4}+\frac{\mathrm{BC}}{2} \cdot \frac{\mathrm{AB}+\mathrm{CD}}{4}$ 


$$
\begin{aligned}
& \Leftrightarrow \frac{\mathrm{AB} \cdot \mathrm{AD}+\mathrm{AB} \cdot \mathrm{BC}}{8}+\frac{\mathrm{CD} \cdot \mathrm{AD}+\mathrm{CD} \cdot \mathrm{BC}}{8}+\frac{\mathrm{AD} \cdot \mathrm{AB}+\mathrm{AD} \cdot \mathrm{CD}}{8}+\frac{\mathrm{BC} \cdot \mathrm{AB}+\mathrm{BC} \cdot \mathrm{CD}}{8} \\
& \Leftrightarrow \frac{\mathrm{AB} \cdot \mathrm{AD}}{8}+\frac{\mathrm{AB} \cdot \mathrm{BC}}{8}+\frac{\mathrm{CD} \cdot \mathrm{AD}}{8}+\frac{\mathrm{CD} \cdot \mathrm{BC}}{8}+\frac{\mathrm{AD} \cdot \mathrm{AB}}{8}+\frac{\mathrm{AD} \cdot \mathrm{CD}}{8}+\frac{\mathrm{BC} \cdot \mathrm{AB}}{8}+\frac{\mathrm{BC} \cdot \mathrm{CD}}{8}
\end{aligned}
$$

donde concluimos $A_{P}$, como:

$$
A_{P}=\frac{\mathrm{AB} \cdot \mathrm{AD}}{4}+\frac{\mathrm{AB} \cdot \mathrm{BC}}{4}+\frac{\mathrm{AD} \cdot \mathrm{CD}}{4}+\frac{\mathrm{BC} \cdot \mathrm{CD}}{4}
$$

donde, também, obtemos a fórmula dada pela expressão 02 .

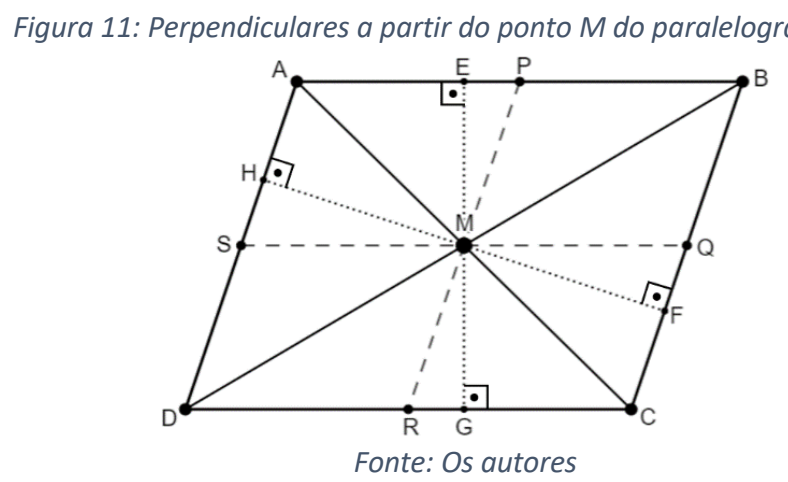

Mas $\mathrm{PM}>\mathrm{EM}, \mathrm{MR}>\mathrm{MG}, \mathrm{SM}>\mathrm{HM}$ e MQ $>\mathrm{MF}$ (figura 11), donde concluímos que a área obtida pelos agricultores, para o paralelogramo, é uma área superestimada.

\section{Quadrilátero qualquer}

É verdade que, nos quadriláteros, os segmentos que unem os pontos médios dos seus lados não verificam expressões 03 e 04, conforme mostraremos adiante. Mas usaremos esta aproximação no intuito de analisar o método empírico:

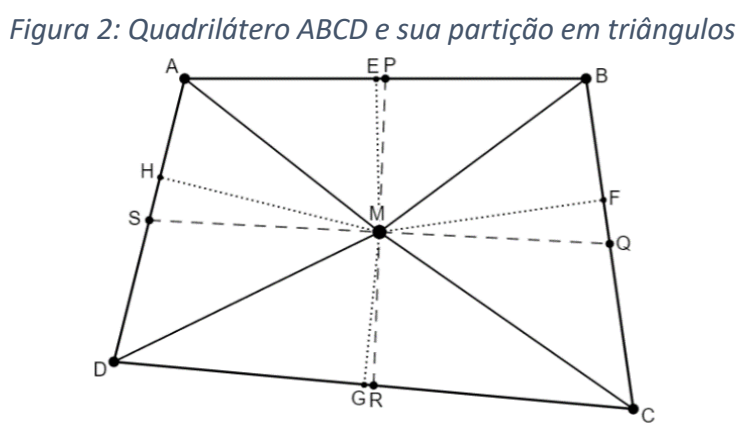

Fonte: Os autores

Assim, calcularemos uma "área", usando como "altura" os segmentos PM, MR, SM e MQ. Assim: 
$A_{Q}=\frac{\mathrm{AB} \cdot \mathrm{PM}}{2}+\frac{\mathrm{CD} \cdot \mathrm{MR}}{2}+\frac{\mathrm{AD} \cdot \mathrm{SM}}{2}+\frac{\mathrm{BC} \cdot \mathrm{MQ}}{2}$

Usando as equações 03 e 04 :

$$
\begin{aligned}
A_{Q} & =\frac{\mathrm{AB}}{2} \cdot \frac{\mathrm{AD}+\mathrm{BC}}{4}+\frac{\mathrm{CD}}{2} \cdot \frac{\mathrm{AD}+\mathrm{BC}}{4}+\frac{\mathrm{AD}}{2} \cdot \frac{\mathrm{AB}+\mathrm{CD}}{4}+\frac{\mathrm{BC}}{2} \cdot \frac{\mathrm{AB}+\mathrm{CD}}{4} \\
& \Leftrightarrow \frac{\mathrm{AB} \cdot \mathrm{AD}+\mathrm{AB} \cdot \mathrm{BC}}{8}+\frac{\mathrm{CD} \cdot \mathrm{AD}+\mathrm{CD} \cdot \mathrm{BC}}{8}+\frac{\mathrm{AD} \cdot \mathrm{AB}+\mathrm{AD} \cdot \mathrm{CD}}{8}+\frac{\mathrm{BC} \cdot \mathrm{AB}+\mathrm{BC} \cdot \mathrm{CD}}{8} \\
& \Leftrightarrow \frac{\mathrm{AB} \cdot \mathrm{AD}}{8}+\frac{\mathrm{AB} \cdot \mathrm{BC}}{8}+\frac{\mathrm{CD} \cdot \mathrm{AD}}{8}+\frac{\mathrm{CD} \cdot \mathrm{BC}}{8}+\frac{\mathrm{AD} \cdot \mathrm{AB}}{8}+\frac{\mathrm{AD} \cdot \mathrm{CD}}{8}+\frac{\mathrm{BC} \cdot \mathrm{AB}}{8}+\frac{\mathrm{BC} \cdot \mathrm{CD}}{8}
\end{aligned}
$$

E, a área $A_{Q}$, é dada por:

$$
A_{Q}=\frac{\mathrm{AB} \cdot \mathrm{AD}}{4}+\frac{\mathrm{AB} \cdot \mathrm{BC}}{4}+\frac{\mathrm{AD} \cdot \mathrm{CD}}{4}+\frac{\mathrm{BC} \cdot \mathrm{CD}}{4}
$$

e, da mesma forma, também obtemos a fórmula dada pela expressão 02.

No caso de um quadrilátero qualquer, temos um caso interessante. Durante a aproximação, existe a medida das alturas dos triângulos AMB, CMD, AMD e BMC, uma vez que é feita para mais e outra vez é feita para menos:

\section{$\mathbf{1}^{\circ}$ análise: erro cometido para cima}

A área empírica considera altura dos triângulos ABM, CDM, os segmentos PM e MR; e como altura dos triângulos ADM, BCM, os segmentos SM e MQ, que são calculados por meio das expressões 03 e 04 .

No entanto, as alturas reais dos triângulos como ABM, CDM, ADM, BCM são EM, MG, HM e MF ao invés de PM, MR, SM e MQ, respectivamente.

Mas $\mathrm{PM}>\mathrm{EM}, \mathrm{MR}>\mathrm{MG}, \mathrm{SM}>\mathrm{HM}$ e MQ $>\mathrm{MF}$ donde concluímos que a área obtida pelos agricultores é uma área superestimada, erro esse que já fora comentado no item anterior.

$\mathbf{2}^{\mathbf{0}}$ análise: erro cometido para baixo

A área do quadrilátero $\mathrm{ABCD}$ é obtida somando-se as áreas dos triângulos, $\mathrm{AMB}, \mathrm{CMD}$, AMD e BMC.

No cálculo da área empírica, consideramos:

$$
\mathrm{SQ}=\frac{\mathrm{AB}+\mathrm{CD}}{2}
$$

$\mathrm{e}$

$$
\mathrm{PR}=\frac{\mathrm{AD}+\mathrm{BC}}{2}
$$


(Basta ver que nas expressões 03 e 04, M é o ponto médio de SQ e PR).

No entanto, se considerarmos a figura 13, e aplicando o estudo de segmentos orientados em geometria analítica, temos que $\mathrm{SS}_{1} \equiv \mathrm{AD}_{1}$. E então

$$
\mathrm{SS}_{1} \equiv \mathrm{AB}+\mathrm{BD}_{1}
$$

Como em um triângulo qualquer, temos que um lado qualquer é sempre menor que a soma dos outros dois lados, temos que:

$$
\mathrm{SS}_{1}<\mathrm{AB}+\mathrm{BD}_{1}
$$

Temos também que, como os quadriláteros $A B C D$ e $A_{1} C B D_{1}$ são semelhantes, $C D=$ $\mathrm{BD}_{1}$. Pela semelhança dos quadriláteros também temos que $\mathrm{SQ}=\mathrm{QS}_{1}$. É verdade que, se $\mathrm{SQ}=$ $\mathrm{QS}_{1}$, então $\mathrm{SS}_{1} \equiv \mathrm{SQ}+\mathrm{QS}_{1}$. Assim:

$$
\mathrm{SS}_{1} \equiv 2 \cdot \mathrm{SQ}
$$

Agora, substituindo a expressão 10 na inequação 09, temos:

$$
\mathrm{SQ}<\frac{\mathrm{AB}+\mathrm{CD}}{2}
$$

Ver figura abaixo:

Figura 3: Quadrilátero $A B C D$ e seu semelhante adjacente $A 1 C B D 1$

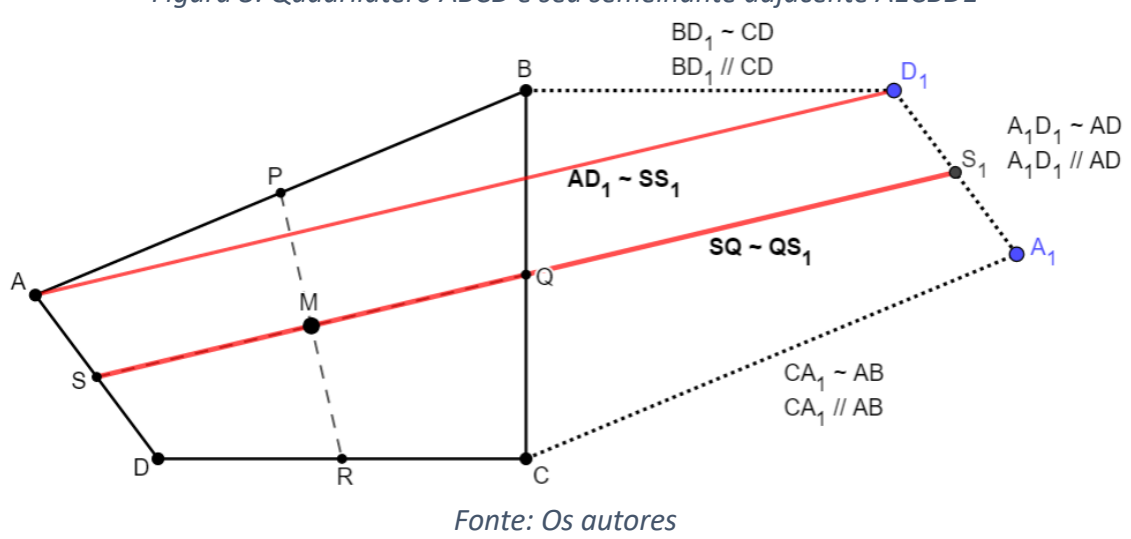

De forma análoga, temos:

$$
\mathrm{PR}<\frac{\mathrm{AD}+\mathrm{BC}}{2}
$$

Neste caso, as medidas SM e MQ que usamos para calcular altura dos triângulos AMD, BMC, são derivadas da média aritmética da inequação 11. Também, as medidas PM e MR, para calcular altura dos triângulos ABM e CDM, são derivadas da média aritmética das inequações 
12, sendo medidas subestimadas. E, neste caso, a área se torna menor do que teríamos se valesse a igualdade.

Esse balanço, para mais e para menos, faz com que a área encontrada empiricamente não se afaste tanto da área real.

\section{Considerações Finais}

Neste trabalho consideramos um método empírico de cálculo de áreas. O método empírico é comum na zona rural e julgamos um conhecimento importante para o meio social em que os agricultores estão inseridos. Acreditamos que dar importância ao conhecimento tradicional e ajudar a preservá-los é ajudar a preservar a cultura de um povo, bem como um incentivo ao desenvolvimento educacional dos filhos das pessoas dessa comunidade.

Em relação aos resultados numéricos de nossa pesquisa, obtidos a partir da questão aberta, verificamos que as áreas obtidas pelo método empírico, fornecidas pelas expressões 03 e 04, forneceram medidas exatas ou aproximadas. Vimos que essas variações dependeram do formato da figura geométrica que representa a região em questão.

A partir de um quadrado, as modificações como dilatação, cisalhamento, modificações aleatórias para os diferentes lados, o método empírico fornece uma área superestimada da área verdadeira do quadrilátero. Configura-se que, quanto maior a deformação na forma dos retângulos, mais a área é distante da área real, erro esse, no entanto, sempre para cima. Assim, esse método só é válido, efetivamente, para formas retangulares.

No último formato geométrico em análise, um quadrilátero qualquer, medidas experimentais sugerem que o valor obtido pelo método empírico também fornece medidas superestimadas do verdadeiro valor. Mesmo para esse caso, a fórmula empírica nos dá uma ideia de que os resultados obtidos são uma aproximação da área real.

Dois pontos importantes precisamos indicar e evidenciar: o primeiro é sobre o fato de não ter sido feita uma análise matemática de que os valores serão sempre superestimados, e o segundo é sobre a existência de uma relação matemática entre as modificações na forma do quadrilátero e a variação no valor da área do quadrilátero. Sabendo que é impossível esgotar tudo em uma pesquisa inicial sobre um tema, deixamos esta lacuna para possíveis estudos posteriores sobre o tema.

Para finalizar, é nítido perceber que o desenvolvimento matemático pode ser expressado de diversas maneiras para se chegar a um resultado. É importante frisar que o método pesquisado tem uma grande relevância dentro da sociedade, pois induz ao indivíduo praticar a matemática e também passar para os seus descendentes. Nesse contexto, percebe-se que o 
quanto a matemática é importante para o desenvolvimento humano e social de uma determinada cultura.

\section{Referências}

D’AMBRÓSIO, U. Etnomatemática: elo entre as tradições e a modernidade. Coleção Tendências em Educação Matemática. 2. ed. Belo Horizonte: Autêntica, 2005.

EVES, H. Introdução à história da matemática. Trad. Hygino H. Domingues. 3. ed. Campinas, SP: Editora UNICAMP, 2004.

EVES, H. Introdução à História da Matemática. Campinas, SP: Editora da Unicamp, 2011.

GARBI, Gilberto Geraldo. A Rainha das Ciências. Um passeio histórico pelo maravilhoso mundo da matemática. São Paulo: Livraria da Física, 2008.

GIL, A. C. Métodos e técnicas de pesquisa social. 5. ed. São Paulo: Atlas, 1999.

GIL, A. C. Como elaborar projetos de pesquisa. 4. ed. São Paulo: Atlas, 2007.

LEONARDO, Fábio Martins de. Projeto araribá, obra coletiva - matemática. São Paulo: Moderna, 9(3), 2010.

MINAYO, Maria Cecília de Souza (org.). Pesquisa Social. Teoria, método e criatividade. 18 ed. Petrópolis, RJ: Vozes, 2001.

MIRANDA, Daniel. Matemática Empírica. Universidade Federal do ABC, 2009.

Disponível em: < http://hostel.ufabc.edu.br/ daniel.miranda/upload/2009/09/ecm1.pdf>. Acesso em: 28 jul. 2020.

OLIVEIRA, Jeanderson. Matemática, Geometria, Área das Figuras Planas. 2012. Disponível em: <www.sosmatematica.blogspot.com.br. > Acesso em: 14 abr. 2020.

ROSA NETO, R. Didática da matemática. São Paulo: Ática, 2002.

SILVA, J. R. N. do. Etnomatemática - abordagem dos diversos tipos de unidades de medidas e sua utilização no sertão alagoano. Maceió. 2016. Disponível em:

http://www.repositorio.ufal.br/handle/riufal/2347

TARTUCE, T. J. A. Métodos de pesquisa. Fortaleza: UNICE - Ensino Superior, 2006. Apostila. 
